\title{
Measuring the fabric evolution of particulate media during load reversals in triaxial tests
}

\author{
Max Wiebicke ${ }^{1,2, *}$, Edward Andò ${ }^{2}$, Ivo Herle $^{1}$, and Gioacchino Viggiani ${ }^{2}$ \\ ${ }^{1}$ Institute for Geotechnical Engineering, Technische Universität Dresden, D-01062 Dresden, Germany \\ ${ }^{2}$ Univ. Grenoble Alpes, CNRS, Grenoble INP, 3SR, F-38000 Grenoble, France
}

\begin{abstract}
The behaviour of granular materials upon load reversal is not yet fully understood. In order to experimentally reveal the microstructure of a specimen subjected to load-unload cycles, a triaxial compression test on lentils is carried out in the x-ray scanner. Before analysing the acquired tomographies, a benchmark analysis is conducted to validate the image analysis tools that are used to extract the fabric from these images. The contact fabric evolves strongly with the shearing in the experiment on lentils. However, only very slight changes of the anisotropy within the cycles are observed.
\end{abstract}

\section{Introduction}

Numerous studies have shown that the fabric of granular materials plays a fundamental role in its macroscopic behaviour. Due to technical limitations this fabric remained inaccessible in real experiments until recently. Thus, the research concentrated on either two-dimensional experiments [1] or post-mortem analyses of real specimen [2]. Discrete element simulations [3], on the other hand, served as a numerical tool to access the grain scale.

With the increasing use and capability of x-ray tomography in the mechanics of granular materials, the grain scale of real three-dimensional specimen finally became accessible. Special apparatus allow to acquire tomographies during a loading process. Determining the fabric from X-ray tomographies remains relatively challenging due to various inherent imaging properties, but recent developments improved the quantitative determination of fabric.

In this contribution we investigate the evolution of fabric due to load reversals. Load reversals lead to abrupt changes of the stiffness of granular materials, e.g., the stiffness increases whenever the loading is reversed. In [4] the fabric evolution in a triaxial test with load reversals has been investigated using discrete element simulations. It remains to be shown whether a similar behaviour can be measured from experiments on real granular materials. Another open question is whether the changes in fabric are sufficiently strong to be measured quantitatively considering the accuracy of these measurements [5].

At first, a benchmark study on the measurement of fabric changes in experiments with load reversals is carried out. Following the benchmark presented in [6] a series of synthetic, but realistic images is created from simulations with the discrete element method (DEM). DEM serves as the ground truth and enables a quantification of the accu-

*e-mail: max.wiebicke@tu-dresden.de racy of the image analysis tools that are to be used on real tomographies.

To investigate the evolution of contact fabric in a real experiment, a triaxial compression test with several unloading and reloading cycles was conducted in the x-ray scanner in Laboratoire 3SR in Grenoble. Here, we present preliminary results of the image analysis of the tomographies acquired during the experiment.

\section{A numerical benchmark}

In [6] we presented a benchmarking strategy to evaluate image analysis tools regarding their accuracy for measuring contact fabric. The example chosen was a monotonic axisymmetric compression test. As the aim of this study is the measurement of contact fabric and its changes during load reversals, another benchmark is carried out.

For this purpose, a discrete element simulation is carried out with the software woodem [7]. The boundaries in this simulation are periodic and a simple linear (Cundall) contact model with the following parameters is used: Young's modulus $E=50 \cdot 10^{6} \mathrm{kPa}$, a ratio between normal and tangent stiffness of $k_{t} / k_{n}=0.4$ and a friction angle of $\tan \varphi=0.4$. A linear particle size distribution between (3.2 and 4.8$) \cdot 10^{-4} \mathrm{~m}$ is chosen.

The sample of 5522 spheres is isotropically compressed to a pressure of $50 \mathrm{kPa}$. It is then compressed in the axial direction at constant radial stress. The loading is reversed until the isotropic stress state is reached and reloaded at several stages of the shearing. At chosen states, mainly when the loading direction is changed, the fabric of the specimen (positions of the spheres and contact orientations) is recorded. The macroscopic behaviour of the specimen is shown in Figure 1.

As in [6], images are created from these states using Kalisphera [8], a tool to create images of spheres mathematically correct regarding the partial volume effect [9]. 

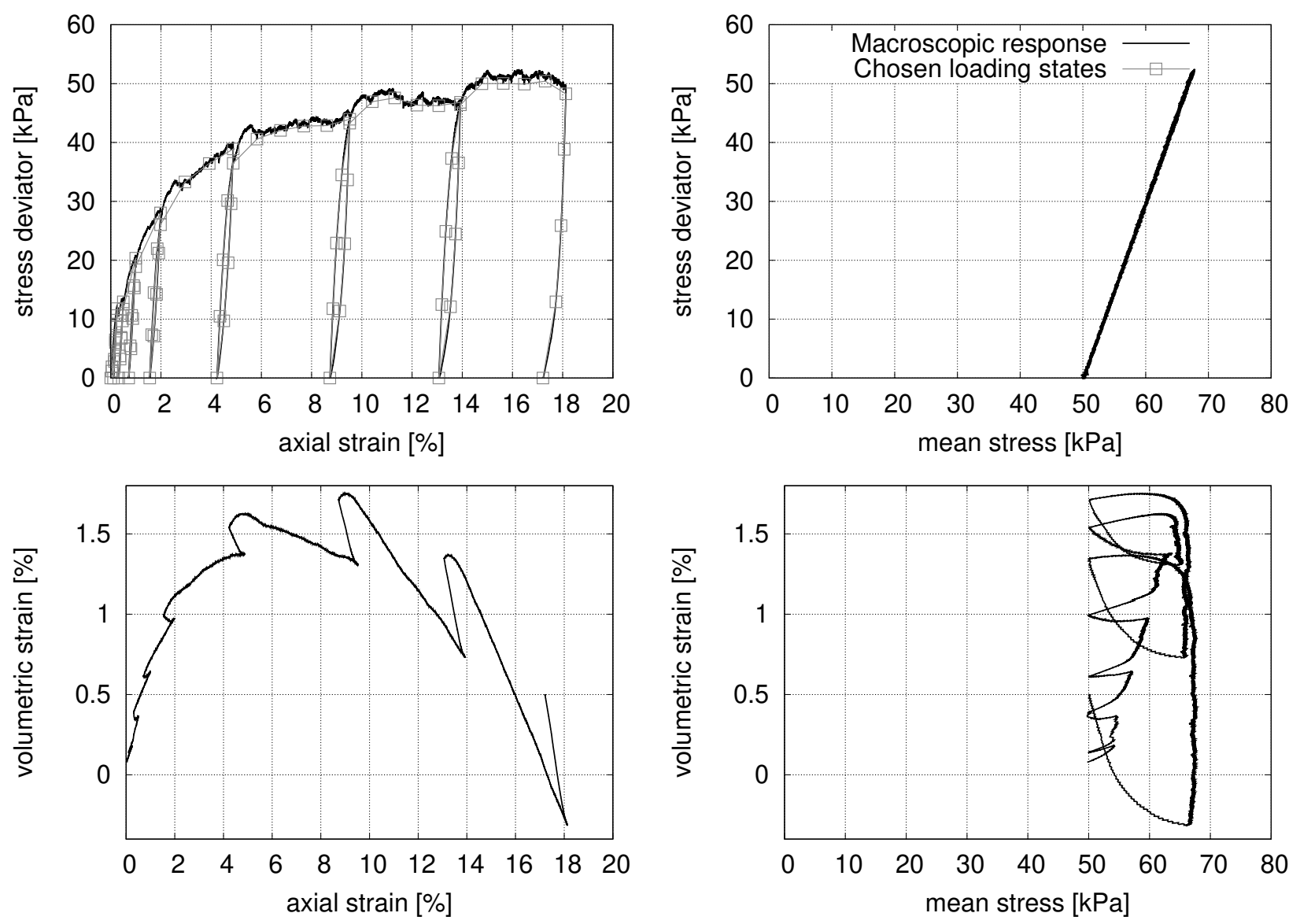

Fig. 1. Macroscopic behaviour of the axisymmetric compression test of the DEM simulation. The chosen states for the image analysis are indicated by the grey squares

Inherent image deficiencies such as noise and blur are applied to the images to obtain realistic representations of real tomographies, see [5] for further details. The image analysis is carried out with the open source software spam [10]. All analysis tools developed in [5] are implemented in spam. The grey-scale images are binarised first and then initially segmented with a VTK watershed. Contacts are detected in the labelled image and the systematic overdetection of contacts happening for grains of non-angular shape is addressed with a local refinement. The orientations are then calculated using the random walker segmentation [11] on each pair of contacting grains. See [5] for more details.

In this small study, we focus on the evolution of the anisotropy of the fabric tensor. The common second order fabric tensor $\mathbf{N}$ [12] is chosen to statistically capture the contact orientations $\mathbf{0}$ :

$$
\mathbf{N}=\frac{1}{C} \sum_{\alpha=1}^{C} \mathbf{o}^{\alpha} \otimes \mathbf{o}^{\alpha}
$$

with $C$ being the number of orientations. The deviator of this tensor is calculated by:

$$
\mathbf{D}=\frac{15}{2}\left(\mathbf{N}-\frac{1}{3} \mathbf{I}\right)
$$

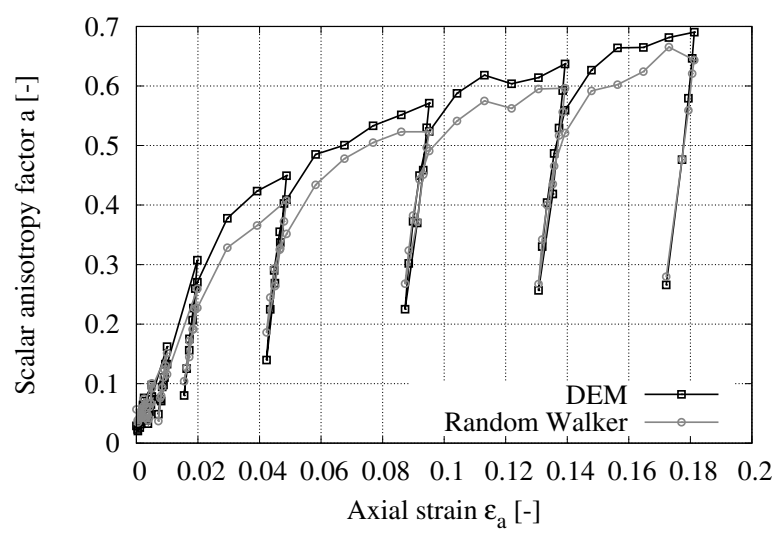

Fig. 2. Evolution of the scalar anisotropy factor. Comparison between the ground truth of the simulation and the results of the image analysis.

with I being the identity tensor. A scalar anisotropy value $a$ can be calculated from [13]:

$$
a=\sqrt{\frac{3}{2} \mathbf{D}: \mathbf{D}}
$$




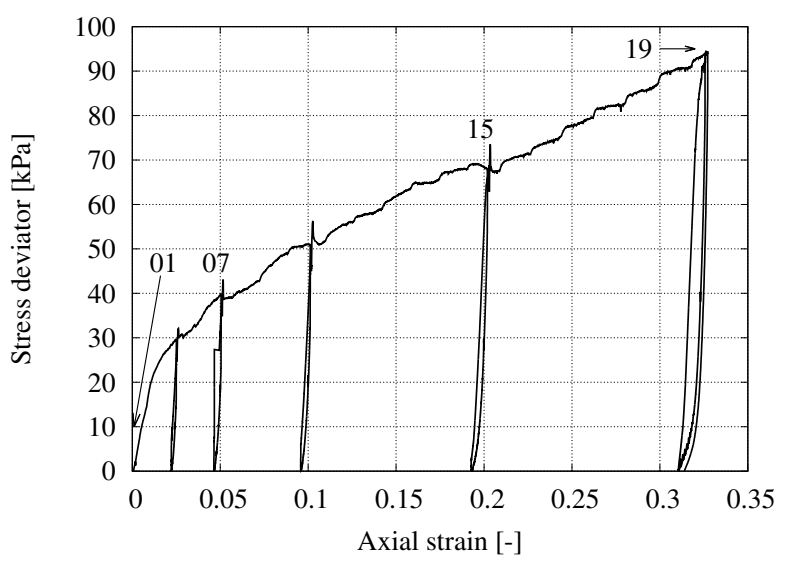

Fig. 3. Stress-strain behaviour of the triaxial compression test on lentils. The identifiers mark the states that are analysed further.

The evolution of the scalar anisotropy factor in both, the reference DEM simulation and the image analysis, is plotted in Figure 2. With ongoing shearing the anisotropy is increasing as expected and shown in [6]. Upon reversing the loading direction, the anisotropy experiences a serious drop - at low strains the initial anisotropy is almost fully recovered, whereas at larger strains the anisotropy is not decreasing until its initial value. The results of the image analysis agree qualitatively to the reference and are only slightly lower in value. With these results, we expect to qualitatively and quantitatively capture the anisotropy in real experiments with load reversals.

All data necessary to carry out and repeat this benchmark or test other image analysis tools on the synthetic images is available in an open access repository [14].

\section{Triaxial compression test on Lentils}

The experiment presented in this study is a triaxial compression test on Lentils. Lentils are chosen as part of a bigger study on the effect of initial anisotropy on the macroscopic as well as microscopic behaviour. The study, however, will not be discussed in this contribution.

The lentils are spooned into a mould in a way to have a $45^{\circ}$ angle with respect to the axial direction in the final specimen. The dry specimen is then isotropically compressed to a pressure of $50 \mathrm{kPa}$. Similarly to the DEM simulation, the specimen is then sheared to chosen states. At these states, the loading direction is reversed until the initial isotropic stress state is almost reached and the specimen is reloaded again. Figure 3 shows the stress-strain behaviour of the specimen during the experiment.

The image analysis is carried out as described above for the synthetic images from DEM using the image analysis software spam. The evolution of contact as well as particle orientations is depicted in Figure 4 as Lambert azimuthal projection plots for the states marked in Figure 3 . The particle orientations are calculated from the moment of inertia and its eigenvectors. The eigenvector corresponding to the largest eigenvalue represents the smallest representative length of the particle. Due to the shape of lentils, this eigenvector is considered to be the significant particle orientation and plotted in Figure 4. The particle fabric agrees well with the fabric that was targeted in the preparation of this specimen, an inherent $45^{\circ}$ angle towards the vertical direction. The contact fabric is more spread, but also aligned to this targeted initial anisotropy. The wider spread is to be expected as the contacts have to provide stability in other directions than only the particle orientation. With ongoing shear the particle and contact orientations realign towards the major principal stress direction, i.e. the centre of the projection plots, but do not reach it at the highest applied shear strain.

The anisotropy as defined in equation (3) expresses the spread of the orientations of either contacts or particles. If the fabric is isotropically distributed, the anisotropy is zero, but in the extreme case it can obtain values of up to 7.5 if all orientations are pointing in the same direction. The evolution of the contact and particle fabric anisotropy is shown in Figure 2. As observed in Figure 4 the specimen inherently has a high initial anisotropy. In the first loading stage the contact anisotropy drops slightly, but with ongoing shear, this anisotropy is increasing, which means that the orientations are concentrating even further in one direction. In the first load-unload cycles, the anisotropy stays almost constant but for the short spike at 0.05 axial strain. The latter cycles show a slight change of contact anisotropy that is increasing at increasing axial strain. This change, however is much smaller than the change in the DEM simulations from the benchmark simulation or [4]. The striking differences is the particle shape as well as the strong inherent anisotropy. It appears that the fabric is locked, which might be explained by the strong and inherent alignment of the lentils that prevents particles to realign. This locked state in terms of particle orientations is observed from the evolution of particle anisotropy in Figure 5. The anisotropy of the particle orientations stays almost constant upon unloading for the low strain as well as for the higher strain cycles.

Contact and particle anisotropy evolve with a highly similar slope. The main difference is the magnitude of the actual values, but the range of change and thus, the rate of change is identical. This is in strong accordance with the findings from 2D DEM simulations on ellipses in [15 $]^{1}$.

\section{Conclusion and perspectives}

In this contribution, we present a preliminary analysis of a triaxial compression test on lentils regarding the fabric evolution due to unloading-reloading cycles. To benchmark the image analysis tools that should be used to extract the contact fabric from the real tomographies, a series of realistic, synthetic images was created on the basis of a DEM simulation. The image analysis of the synthetic images agrees qualitatively and quantitatively to the reference DEM simulation.

The experiment is prepared to have a strong inherent anisotropy with particles oriented $45^{\circ}$ to the vertical di-

\footnotetext{
${ }^{1}$ It should be noted that our principal particle orientation is the short axis in contrast to [15], in which the longest axis was used as the reference particle orientation.
} 


\section{Particle orientations}

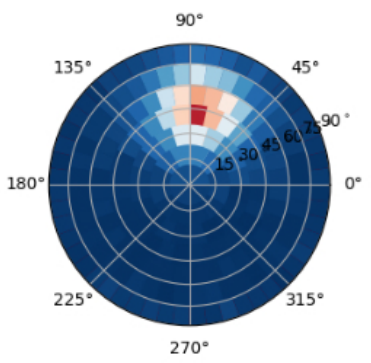

01

(initial)

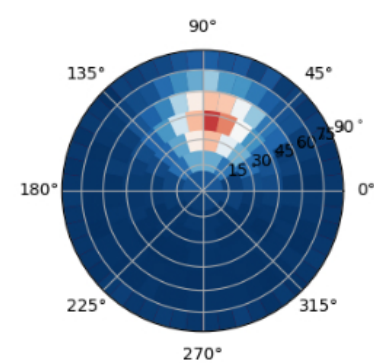

07

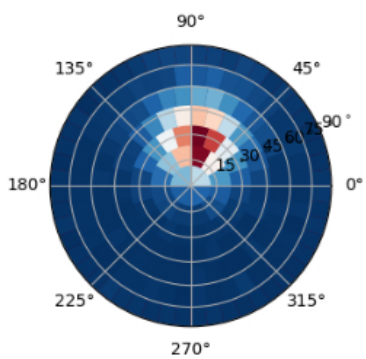

15

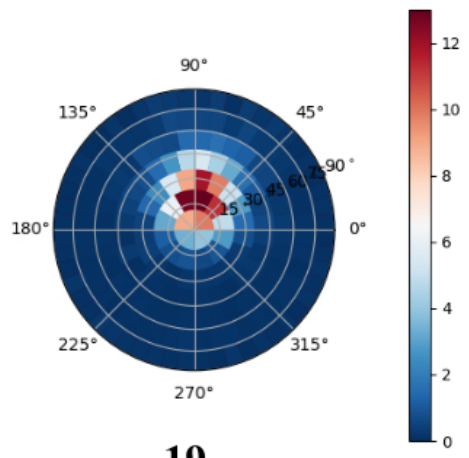

19

(highest shear strain)

\section{Contact orientations}
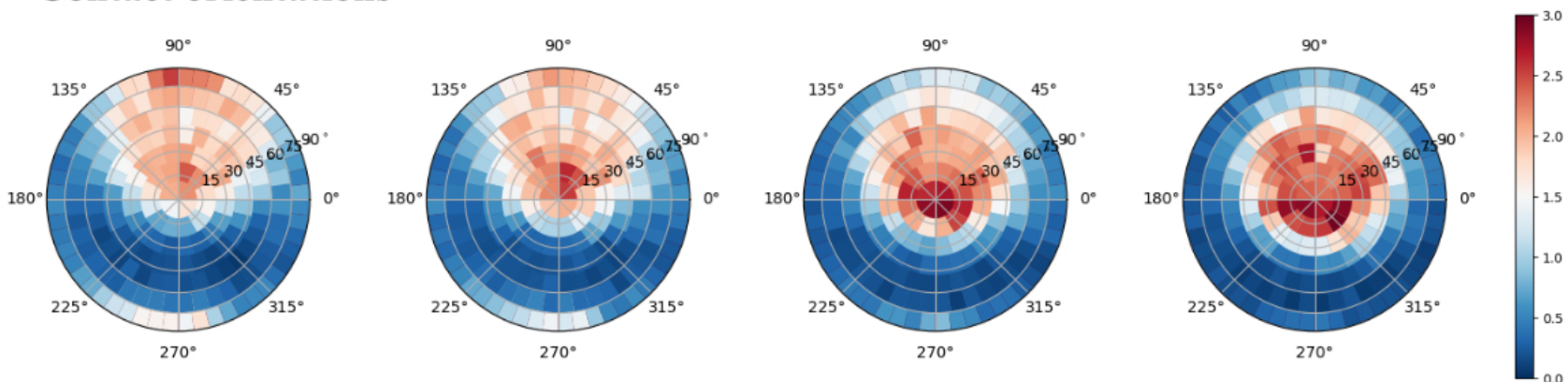

Fig. 4. Lambert azimuthal equal are plots of particle and contact orientations at several states of the loading. The center of the image is the axial direction whereas the outer ring represents the axial plane. The orientations in each bin are normalised by the average orientations per bin.

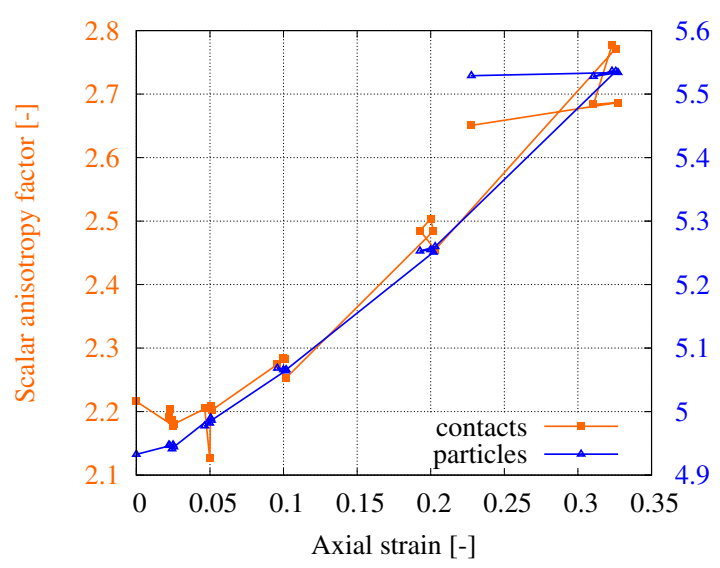

Fig. 5. Evolution of anisotropy of contact and particle fabric in the triaxial compression test on lentils.

rection. This initial anisotropy is increased further with shearing as the principal fabric direction aligns with the major principal stress. A serious change of fabric within the unload-reload cycles cannot be observed. A possible reason can be a locking of the fabric due to the strong inherent anisotropy and the similar particle shape of all particles. Another reason for the observations could be the fabric tensor we chose. Employing a different normalisation of the fabric tensor and/or a higher degree of ap- proximation might show a different behaviour. Especially, at higher macroscopic shear strains, deformation localised in the specimen. An analysis extracting subsets might reveal a different and/or more pronounced fabric evolution. Subsets inside and outside the zones of localised deformation are to be extracted and the fabric will be calculated in these.

\section{References}

[1] K. Wiendieck, Die Bautechnik 6, 196 (1967)

[2] M. Oda, Soils and Foundations 12 (1972)

[3] P.A. Cundall, O.D.L. Strack, Géotechnique 29, 47 (1979)

[4] C. O’Sullivan, L. Cui, Powder Technology 193, 289 (2009)

[5] M. Wiebicke, E. Andò, I. Herle, G. Viggiani, Measurement Science and Technology 28, 124007 (2017)

[6] M. Wiebicke, V. Šmilauer, I. Herle, E. Andò, G. Viggiani, Validation of Synthetic Images for Contact Fabric Generated by DEM, in Proceedings of ChinaEurope Conference on Geotechnical Engineering (2018), ISSN 1866-8755

[7] V. Šmilauer, Woo Documentation (2016), https://woodem.org

[8] A. Tengattini, E. Andò, Measurement Science and Technology 26 (2015) 
[9] S. Weis, M. Schröter, Review of Scientific Instruments (2017), 1612.06639

[10] E. Andò, R. Cailletaud, E. Roubin, O. Stamati, the spam contributors, spam: The software for the practical analysis of materials, https://ttk. gricad-pages.univ-grenoble-alpes.fr/spam/ (2017-)

[11] L. Grady, Random walks for image segmentation, in IEEE Transactions on Pattern Analysis and Machine Intelligence (2006), Vol. 28

[12] K.I. Kanatani, International Journal of Engineering Science 22, 149 (1984)

[13] X. Gu, J. Hu, M. Huang, Granular Matter 19, 1 (2017)

[14] M. Wiebicke, Benchmark analysis of synthetical images - source code and example dem data, http: //dx.doi.org/10.25532/OPARA-14

[15] P. Fu, Y. Dafalias, International Journal for Numerical and Analytical Methods in Geomechanics 35, 1918 (2011) 